\title{
INVESTIGACIONES
}

\section{Dinamización tecnológica de la escuela a través del liderazgo del coordinador TIC"}

\author{
Technological dynamism of school through the leadership of the ICT coordinator
}

\author{
Alicia González-Pérez \\ Facultad de Formación del Profesorado, Universidad de Extremadura, España \\ Telf.: (34) 927257972. Correo electrónico: aliciagp@unex.es
}

\begin{abstract}
RESUMEN
Esta investigación tiene como objetivo analizar los factores que tienen mayor incidencia en la dinamización tecnológica de los centros innovadores seleccionados en las cuatro comunidades autonómicas participantes del estudio y si existen diferencias entre ellas. Los datos se han recogido en una muestra de 49 centros, a través de una escala elaborada para tal objetivo y que se ha dirigido a los coordinadores TIC y al equipo directivo de los centros seleccionados. Una vez que se ha comprobado que la escala es válida y fiable se han realizado análisis descriptivos básicos y se ha aplicado la técnica de análisis ANOVA. Aunque no se encontraron diferencias significativas entre las comunidades estudiadas, este estudio ha proporcionado datos cuantitativos muy interesantes sobre las acciones que el coordinador TIC pone en práctica. Además, también son discutidas las implicaciones que el liderazgo tiene en el rol que el coordinador TIC juega en la escuela.
\end{abstract}

Palabras clave: tecnología educativa, escuelas innovadoras, integración tecnológica, director de centro.

\begin{abstract}
The main aim of this research is to analyze factors with the greatest impact on the technological dynamism in certain schools. As well, the differences between Autonomous Communities are going to be researched through the ANOVA technique. To determine the sample, a selection process was devised to provide the most useful data, which was collected in a sample of 49 schools through a scale elaborated and directed to ICT coordinators and managers from the schools selected. After proving the scale, a validity and reliability test was performed to examine the internal consistency, means and standard deviations were calculated and the ANOVA technique was applied. Although significant differences were not found between Autonomous Communities, this study has provided a quantitative measure of actions that ICT coordinators assumed in practice. The implications of leadership in the role of ICT coordinators in schools were also discussed.
\end{abstract}

Key words: educational technology, innovative Schools, technology integration, headmaster.

Esta investigación se enmarca en una de las actuaciones desarrolladas dentro del Proyecto I+D+i. "Políticas educativas autonómicas y sus efectos sobre la innovación pedagógica apoyada en la integración de las TIC en los centros escolares". Ministerio de Ciencia e Innovación. Dirección General de Programas y Transferencia del Conocimiento. Ref. SEJ200612435-C05-05/EDUC. Gobierno de España. 


\section{INTRODUCCIÓN}

Los responsables políticos, así como los agentes educativos reconocen, sin duda, que las TIC (Tecnologías de la Información y la Comunicación) son un elemento fundamental para alcanzar la transformación de la educación y de la formación. Es por ello que las TIC son un elemento clave para la innovación y la creatividad, y para el aprendizaje en general. Sin embargo, todo el potencial formador y educativo que tienen las TIC no se ve suficientemente reflejado en la educación formal.

En España en los últimos veinte años se han ido sucediendo un conjunto de planes y programas que han tenido como objetivo el desarrollo tecnológico de las escuelas, la formación en TIC del profesorado y la integración de las TIC en los procesos de enseñanza y aprendizaje, fundamentalmente. Sin embargo, esta estrategia queda congelada cuando en marzo de 2012 se suspende el Programa Escuela 2.0 'un ordenador por niño'.

En esta sucesión de programas ha habido una inversión creciente destinada a hacer extensivo el uso de las TIC en los centros docentes. Por ello, se hace necesario investigar sobre el éxito o fracaso de la implementación de las TIC en el ámbito escolar y su relación con el papel que juegan los coordinadores TIC y los directivos de un centro para la dinamización tecnológica. Así pues, este estudio tendrá en cuenta los datos obtenidos en las comunidades participantes en la investigación (Andalucía, Extremadura, Canarias y País Vasco) y el grado en que se incentiva el uso de las TIC en la docencia, la formación permanente del profesorado en TIC, la creación de ambientes estimulantes para la innovación con TIC, la creación de grupos de trabajo entre el profesorado que usa las TIC, la participación del profesorado en proyectos de innovación TIC, la comunicación con las familias a través de las TIC y su formación.

\section{EL COORDINADOR TIC COMO PIEDRA ANGULAR DEL CAMBIO TECNOLÓGICO DE UN CENTRO}

La búsqueda de condiciones que apoyen la integración de la tecnología en las aulas es un tema de especial interés para investigadores (Fishman \& Zhang, 2003), diseñadores de políticas (Zhao \& Conway, 2001) y profesionales encargados de la planificación tecnológica, referida esta al proceso de desarrollo, implementación y revisión de un plan que guie a los responsables de una organización en la dinamización tecnológica del centro.

Una de las acciones de interés en el campo de las TIC es la búsqueda de condiciones que apoyen la integración y el uso de las TIC en el aula y el centro, ya que se observa una brecha entre estos dos eslabones (Tondeur, van Braak \& Valcke, 2007).

Aunque no se ha sistematizado de forma específica cómo las escuelas, como contextos específicos, implementan sus proyectos TIC, los investigadores en este tema son conscientes de la importancia de diseñar e implantar un proyecto TIC que esté basado en las necesidades de la escuela, donde se facilite la integración y uso de las TIC en todos sus niveles.

Las escuelas como organizaciones educativas han de entender el potencial que tienen las TIC y la complejidad de su aplicación en términos de innovación y cambio (Lai, Trewern \& Pratt, 2002; Tearle, 2004). Como destacan Strudler, Falba y Hearrington (2001) el cambio educativo con TIC es algo permanente que exige coordinación y apoyo continuo. Por ello, las escuelas precisan de un facilitador o agente de cambio que pueda orientar 
y apoyar al centro durante el proceso de implementación de las TIC en la educación. (Watson, 2006).

Así pues, los coordinadores TIC, como agentes que regulan los ritmos de desarrollo e innovación de las escuelas, y los directores de los centros juegan un papel importante en la integración de las TIC en el currículum y en la organización de la actividad del centro. (Devolder et al., 2010; Lai et al., 2002; Marcovitz, 2000). Con buen entendimiento del proceso, el coordinador TIC puede ayudar a cambiar la escuela en el buen sentido (Marcovitz, 2000).

De ahí el interés en conocer cuáles son los aspectos más relevantes acerca de cómo los coordinadores TIC ejercen el liderazgo en la promoción e implementación de las políticas educativas TIC en los centros escolares (De Pablos et al., 2010; González-Pérez, 2010, 2012, 2014a, 2014b; González-Pérez \& De Pablos, 2015) ya que el liderazgo está asociado empíricamente con la mejora de las condiciones profesionales del profesorado, así como de los aprendizajes de los alumnos (Vanderlinde, Dexter \& van Braak, 2012).

A este respecto se han publicado los resultados de algunas investigaciones que resaltan la necesidad de profesionalizar la figura de coordinador TIC (Pérez, Aguaded \& Fandos, 2010) y de apostar por un cambio en la forma de organizar los recursos tecnológicos para que el profesorado haga un uso de las TIC de forma innovadora. Además, se han asignado al coordinador TIC distintos roles, desde el tecnológico hasta el de líder (Lai \& Pratt, 2004) o el de agente de cambio (Vanderlinde, et al., 2012).

Es de interés destacar algunos estudios previos que han tenido por objetivo dilucidar las funciones del coordinador TIC o su papel en las escuelas de primaria y secundaria a través de la aplicación de métodos cualitativos de investigación, (Fernández \& Lázaro, 2008; Fernández, Sosa \& Garrido, 2011; Lai \& Pratt, 2004; Marcovitz, 2000), métodos cuantitativos (Devolder et al., 2010) o técnicas mixtas (Area, 2010; Pérez et al., 2010).

Hancock (1990) define el rol del coordinador TIC como un tutor para los estudiantes, como un modelo para los maestros y como una persona que apoya estratégicamente al personal administrativo. El papel del coordinador consiste en dar soporte a los estudiantes cuando tienen problemas de hardware o software. El rol que ejerce para los profesores consiste en mostrarles cómo ellos pueden usar los ordenadores para apoyar el proceso instructivo; y como persona estratégica, el coordinador TIC planifica, desarrolla, organiza e implementa actividades para la enseñanza (Devolder et al., 2010).

Sin embargo, en otros estudios se revela que en la práctica los roles predominantes asumidos por los coordinadores TIC incluyen soporte técnico y apoyo a los niños durante su proceso de aprendizaje. En este contexto, las tareas llevadas a cabo por el coordinador incluyen la enseñanza de competencias TIC, la coordinación del plan TIC del centro, la gestión del hardware y el apoyo técnico, y entrenamiento. Además, se enfatiza sobre el rol de liderazgo TIC para facilitar un buen uso de las TIC (BECTA, 2010).

Según Lai y Pratt (2004) las tareas llevadas a cabo por los coordinadores TIC tienen que ver con la asistencia técnica y educativa, principalmente. Estas tareas incluyen la gestión de la red de la escuela y el sitio web (Devolder et al., 2010), así como otras cuestiones que estén contenidas en el proyecto TIC del centro. Lai y Pratt (2004) también hacen hincapié en la necesidad de considerar al coordinador TIC como líder lo que implica que el coordinador debe de tener tiempo suficiente para ejercer dicho rol. Strudler (1994) destaca que el rol de liderazgo es necesario para establecer una visión compartida y desarrollar el proyecto TIC. 
Finalmente se destaca que la asignación de multi-tareas incrementa la complejidad del rol que los coordinadores TIC asumen en la implementación de las TIC en las escuelas. Por tanto, el coordinador TIC debe responder las demandas del profesorado en dos sentidos: el pedagógico y el técnico, además de responder a otras sensibilidades que acojan a toda la comunidad educativa y especialmente a la familia.

\section{OBJETIVOS DE LA INVESTIGACIÓN}

Los objetivos que se persiguen en este estudio son los que se especifican a continuación:

- Analizar qué factores tienen mayor incidencia en la dinamización tecnológica de los centros innovadores seleccionados en las cuatro comunidades autonómicas participantes en el estudio, que tanto directivos como coordinadores TIC apoyan.

- Conocer si existen diferencias significativas entre las Comunidades participantes en el estudio con respecto a los factores identificados que dinamizan la proyección tecnológica de un centro educativo.

\section{DISEÑO METODOLÓGICO}

En esta investigación se ha optado por un estudio tipo encuesta a través de la elaboración de un cuestionario dirigido a los gestores del centro, con el fin de conocer la dinamización tecnológica de los centros escolares de enseñanza no obligatoria en las comunidades de Andalucía, Extremadura, Canarias y País Vasco. Este cuestionario tiene la particularidad de poder ser contestado de manera individual, por el director del centro o el coordinador TIC, o de manera colegida, al considerarse ambos pieza clave para el cambio pedagógico y tecnológico de un centro. Según las conclusiones alcanzadas por Anderson y Dexter (2005) el liderazgo fue considerado un indicador más crítico en la predicción del éxito de la implementación de las TIC que los indicadores referidos al desarrollo de las infraestructuras.

\subsection{MUESTRA E INSTRUMENTO DE RECOGIDA DE DATOS}

La muestra la han conformado únicamente los centros que fueron valorados por los responsables políticos y gestores educativos, como centros innovadores en los que se realizan buenas prácticas con TIC. De ahí que el total de centros seleccionados fue de solo 49 en las cuatro comunidades estudiadas, reflejo del escaso número de centros que fueron valorados como modelos en cuanto al diseño e implantación de innovaciones o buenas prácticas con TIC.

El cuestionario constaba de varias partes bien diferenciadas. El primer apartado se centró en conocer la participación que las comunidades implicadas en el estudio mostraban sobre las políticas educativas TIC. La siguiente sección medía los niveles de conocimiento, aplicación e innovación que tienen las TIC en la gestión del centro, en la práctica docente y en la proyección socioeducativa del centro. Un tercer apartado se centró en conocer el apoyo que los gestores de los centros prestaban a dichas políticas y a la gestión del cambio tecnológico. Posteriormente, se estudiaban las dificultades y los factores facilitadores implicados en el desarrollo de la innovación y las buenas prácticas TIC en el centro, 
cuestiones a tener en cuenta a la hora de diseñar políticas educativas TIC. Finalmente, se abrió un apartado en el cual cada centro podría expresar qué buenas prácticas TIC se estaban desarrollando en su centro.

De la totalidad del cuestionario, en este trabajo, únicamente se presentan los resultados correspondientes a esta escala tipo Likert que tiene como finalidad medir el grado en que los directivos y los coordinadores TIC apoyan la dinamización tecnológica en su centro. En la Tabla 1 se especifican acciones desarrolladas de las cuales se han recogido los datos.

Tabla 1. Escala de acciones desarrolladas por el equipo directivo y el coordinador TIC para la dinamización tecnológica de los centros

\begin{tabular}{|l|l|l|l|l|l|}
\hline $\begin{array}{l}\text { APOYO DE LOS EQUIPOS DIRECTIVOS DE LAS POLÍTICAS } \\
\text { TIC EN EL CENTRO. } \\
\text { (Señale con una x la casilla que más se ajuste a tu puntuación. }\end{array}$ & 1 & 2 & 3 & 4 & 5 \\
1(nada) 2(poco) 3(suficiente) 4(bastante) 5 (mucho).
\end{tabular}

Fuente: elaboración propia.

\subsection{ANÁLISIS ESTADÍSTICOS}

Los resultados obtenidos para dicha escala se han analizado mediante el programa informático SPSS versión 18. Se han aplicado técnicas de estadística descriptiva a través de procedimientos de frecuencias y descriptivos de tendencia central. Posteriormente se ha realizado un análisis de la varianza (ANOVA) para contrastar la hipótesis nula sobre si las medias de las distintas comunidades autónomas son iguales. Previo a estos análisis se realizó una valoración estadística de la validez y fiabilidad de la escala utilizada.

\section{RESULTADOS}

Antes de presentar los resultados obtenidos con respecto a los objetivos de la investigación se aportan los datos de validez y fiabilidad de la escala. Para conocer la validez de constructo se aplicó la medida de adecuación muestral de Kaiser-Meyer-Olkin (KMO) y el test de esfericidad de Barlett, que indican la pertinencia de aplicar la técnica del análisis factorial a los datos. 
Tabla 2. Kaiser-Meyer-Olkin y prueba de Bartlet: Escala de acciones desarrolladas por el equipo directivo y el coordinador TIC para la dinamización tecnológica de los centros

\begin{tabular}{|c|c|c|}
\hline \multicolumn{2}{|c|}{ Medida de adecuación muestral de Kaiser-Meyer-Olkin } & .855 \\
\hline \multirow{3}{*}{ Prueba de esfericidad de Bartlett. } & Chi-cuadrado aproximado & 128.564 \\
\cline { 2 - 3 } & gl & 21 \\
\cline { 2 - 3 } & Sig. & .000 \\
\hline
\end{tabular}

Fuente: elaboración propia.

Como se observa en la Tabla 2, el índice KMO es >.5 lo que indica que la intercorrelación entre variables es grande y esto es indicativo de la existencia de suficiente correlación. Por tanto, la validez interna obtenida es buena.

Seguidamente se procedió a realizar un análisis factorial de la escala, mediante un análisis de componentes principales. Los resultados mostraron que con un factor se explicaba el $62.706 \%$ de la varianza acumulada. Ese componente va dirigido a fortalecer las sinergias de los centros, en cuanto a la formación, comunicación, participación y el uso de las TIC, para que se produzca innovación en los centros. Por tanto, el equipo directivo y el coordinador TIC son una pieza clave del cambio y un punto de inflexión entre las políticas que se dictaminan desde un entorno macro y la práctica educativa que se genera en los centros y las aulas.

Para conocer la fiabilidad de la escala utilizada se aplicó el Alfa de Cronbach. Se obtuvo un Alfa de Cronbach de .888 (Alpha Std.=.900) lo cual significa que la escala global tiene una fiabilidad alta debido a que supera el .80 .

Con respecto al primer objetivo de esta investigación 'analizar qué factores tienen mayor incidencia en la dinamización tecnológica de los centros innovadores seleccionados en las cuatro comunidades autonómicas participantes en el estudio, que tanto directivos como coordinadores TIC apoyan' los resultados se presentan en la Tabla 3.

Tabla 3. Análisis estadísticos de acciones desarrolladas por el equipo directivo y el coordinador TIC para la dinamización tecnológica de los centros

\begin{tabular}{|l|c|c|c|}
\hline \multicolumn{1}{|c|}{$\begin{array}{l}\text { APOYO DE LOS EQUIPOS DIRECTIVOS } \\
\text { DE LAS POLÍTICAS TIC EN EL CENTRO }\end{array}$} & N & Media & $\begin{array}{c}\text { Desv. } \\
\text { típ }\end{array}$ \\
\hline $\begin{array}{l}\text { D38-Fomenta la creación de grupos de trabajo entre el profesorado para el uso } \\
\text { de las TIC }\end{array}$ & 46 & 3.61 & 1.064 \\
\hline D39-Ambiente de trabajo estimulante para la innovación con TIC & 46 & 3.70 & .866 \\
\hline D40-Fomenta la formación permanente del profesorado en TIC & 46 & 3.83 & .973 \\
\hline D41-Facilita la formación de las familias en TIC & 39 & 2.26 & 1.352 \\
\hline D42-Facilita la comunicación de las familias con el centro a través de las TIC & 42 & 3.14 & 1.475 \\
\hline D43-Incentiva el uso de TIC en la docencia & 47 & 3.57 & 1.078 \\
\hline D44-Estimula la participación del profesorado en proyectos de innovación TIC & 43 & 3.42 & 1.118 \\
\hline
\end{tabular}


Si se representaran las medias globales de cada una de las acciones en una gráfica se observaría que todas las variables obtienen valores superiores a la media de la escala (2.5), excepto la que hace referencia a facilita la formación de las familias en TIC. Por tanto, se demuestra que el resto de acciones se consideran parte del quehacer de los equipos directivos y el coordinador TIC en pro de la dinamización tecnológica en los centros. Por tanto, se consideran como acciones relevantes para el éxito de las políticas educativas TIC.

Los factores que tienen mayor incidencia en la dinamización tecnológica son, respectivamente, el fomento de la formación permanente del profesorado en TIC (media=3.83) y la creación de un ambiente de trabajo estimulante para la innovación con TIC (media=3.70). Por el contrario, los factores con menor relevancia son los que se refieren a facilitar la formación de las familias en TIC (media=2.26), seguido de facilitar la comunicación de las familias con el centro a través de las TIC (media=3.14).

Estas respuestas ofrecen una imagen clara y real sobre las dimensiones en las que descansan las políticas educativas TIC. La dimensión formación del profesorado, la creación de espacios estimulantes para el trabajo en grupo, así como el fomento de nuevas metodologías innovadoras, constituyen los pilares fundamentales en los que se apoyan los directivos y los coordinadores TIC encuestados para implementar las políticas educativas TIC en sus centros. De ello, se deduce que se prioriza la formación permanente del profesorado y la creación de espacios que estimulen la creación de grupos de trabajo TIC como dimensiones clave en el éxito de las políticas educativas TIC.

Con respecto al segundo objetivo de esta investigación: conocer si existen diferencias significativas entre las Comunidades participantes en el estudio con respecto a los factores identificados que dinamizan la proyección tecnológica de un centro educativo, se aplica la técnica de análisis ANOVA. Para ello se realizó un análisis de varianza tomando como variables dependientes cada uno de los ítems de la escala y como factor generador de los grupos la variable 'Comunidad Autónoma'. En la Tabla 4 se muestran los resultados obtenidos.

Tabla 4. Resultados obtenidos del análisis de varianza aplicado a las cuatro comunidades autónomas que participan en el estudio

\begin{tabular}{|c|c|c|c|c|c|c|}
\hline \multicolumn{7}{|c|}{$\begin{array}{l}\text { ANOVA. COMUNIDADES AUTONOMAS: } \\
\text { Andalucía, Canarias, Extremadura y País Vasco }\end{array}$} \\
\hline & & $\begin{array}{l}\text { Suma de } \\
\text { cuadrados }\end{array}$ & gl & $\begin{array}{c}\text { Media } \\
\text { Cuadrática }\end{array}$ & $\mathrm{F}$ & Sig. \\
\hline \multirow{3}{*}{$\begin{array}{l}\text { D38. Fomenta la creación de grupos } \\
\text { de trabajo entre el profesorado para } \\
\text { el uso de las TIC }\end{array}$} & Inter-grupos & 8,214 & 3 & 2,738 & 2,690 &, 058 \\
\hline & Intra-grupos & 42,743 & 42 & 1,018 & & \\
\hline & Total & 50,957 & 45 & & & \\
\hline \multirow{3}{*}{$\begin{array}{l}\text { D39. Ambiente de trabajo estimu- } \\
\text { lante para la innovación con TIC }\end{array}$} & Inter-grupos &, 718 & 3 &, 239 &, 305 &, 822 \\
\hline & Intra-grupos & 33,021 & 42 & ,786 & & \\
\hline & Total & 33,739 & 45 & & & \\
\hline
\end{tabular}




\begin{tabular}{|l|l|c|c|l|l|l|}
\hline \multirow{2}{*}{$\begin{array}{l}\text { D40. Fomenta la formación perma- } \\
\text { nente del profesorado en TIC }\end{array}$} & Inter-grupos & 2,125 & 3 &, 708 &, 735 &, 537 \\
\cline { 2 - 8 } & Intra-grupos & 40,484 & 42 &, 964 & & \\
\cline { 2 - 8 } & Total & 42,609 & 45 & & & \\
\hline \multirow{2}{*}{$\begin{array}{l}\text { D41. Facilita la formación de las fa- } \\
\text { milias en TIC }\end{array}$} & Inter-grupos & 5,836 & 3 & 1,945 & 1,071 &, 374 \\
\cline { 2 - 8 } & Intra-grupos & 63,600 & 35 & 1,817 & & \\
\cline { 2 - 8 } & Total & 69,436 & 38 & & & \\
\hline \multirow{2}{*}{$\begin{array}{l}\text { D42. Facilita la comunicación de las } \\
\text { familias con el centro a través de las } \\
\text { TIC }\end{array}$} & Inter-grupos & 2,229 & 3 &, 743 &, 325 &, 807 \\
\cline { 2 - 8 } & Intra-grupos & 86,914 & 38 & 2,287 & & \\
\cline { 2 - 8 } & Total & 89,143 & 41 & & &, 954 \\
\hline \multirow{2}{*}{$\begin{array}{l}\text { D43. Incentiva el uso de TIC en la } \\
\text { docencia }\end{array}$} & Inter-grupos &, 407 & 3 &, 136 &, 110 & \\
\cline { 2 - 8 } & Intra-grupos & 53,082 & 43 & 1,234 & & \\
\cline { 2 - 8 } & Total & 53,489 & 46 & & & \\
\hline \multirow{2}{*}{$\begin{array}{l}\text { D44. Estimula la participación del } \\
\text { profesorado en proyectos de innova- } \\
\text { ción TIC }\end{array}$} & Inter-grupos & 3,882 & 3 & 1,294 & 1,039 &, 386 \\
\hline & Intra-grupos & 48,584 & 39 & 1,246 & & \\
\hline & Total & 52,465 & 42 & & & \\
\hline
\end{tabular}

Fuente: elaboración propia.

Los resultados obtenidos nos indican que no existen diferencias significativas entre comunidades autónomas en cuanto a la dinamización tecnológica que desarrollan los directivos y/o coordinadores TIC. La variable que obtiene una significatividad más próxima al .05 es la que hace referencia a fomenta la creación de grupos de trabajo entre el profesorado que usa las TIC con una significatividad del .058. Por ello, no se rechaza la hipótesis nula de que en todos los grupos las medias son iguales.

\section{DISCUSIÓN}

A pesar de llevar más de dos décadas haciendo grandes esfuerzos económicos en la implementación y uso de las TIC en los centros escolares a través de las administraciones públicas se evidencia que la utilización pedagógica de las TIC todavía no se ha generalizado ni se ha convertido en una práctica integrada en los centros escolares (Area, 2010). La integración y uso de las TIC con fines educativos sigue siendo baja, y muchas de las prácticas docentes no representan un avance, innovación o mejora respecto a las prácticas tradicionales. Balanskat, Blamire y Kefala, (2006) concluyeron en el informe European Schoolnet, bajo el patrocinio de la Comisión Europea, que «los profesores usan 
las TIC para apoyar las pedagogías ya existentes» sin representar una alteración sustantiva de los principios y métodos de enseñanza. Esto nos ha de hacer reflexionar sobre qué mecanismos pueden ayudar a dinamizar el desarrollo tecnológico en una escuela para que tenga implicaciones pedagógicas más exitosas.

Afshari, Bakar, Su-Luan y Siraj (2012) destacan que para que las tecnologías sean integradas e implementadas efectivamente en la escuela han de ser apoyadas particularmente por los que ejercen el liderazgo en las escuelas. Además, los coordinadores TIC y los directivos han de aprender y usar las TIC en sus tareas instructivas y administrativas, han de apoyar a sus profesores en los procesos de cambio y han de proveer oportunidades de desarrollo para ellos y su personal. Según Schiller (2003) los directores de escuela como facilitadores del cambio tienen la responsabilidad de iniciar e implementar cambios en la escuela a través del uso de las TIC y pueden facilitar la toma de decisiones complejas para integrar las TIC en el aprendizaje, la enseñanza y la organización de la escuela.

De entre las acciones propuestas en la investigación para apoyar la implementación de las políticas TIC en un centro, tanto los coordinadores TIC como los equipos directivos encuestados han considerado de mayor a menor relevancia: fomentar la formación permanente del profesorado, crear ambientes de trabajo estimulantes para innovar con TIC, crear grupos de trabajo donde se usen las TIC, incentivar el uso de las TIC en la docencia, estimular al profesorado a que participe en proyectos de innovación TIC, facilitar la comunicación con las familias a través de las TIC y su formación.

Es cierto que en este estudio no han quedado identificadas diferencias significativas entre las comunidades estudiadas. Sin embargo, según los resultados presentados se considera que, a pesar de que existen diferencias en las políticas educativas TIC autonómicas y en su implantación histórica, existe una cierta similitud y convergencia en cuanto a las actuaciones que los centros desarrollan. Además, según las puntuaciones globales se incide en fomentar la formación permanente del profesorado y la creación de un clima de trabajo estimulante para la innovación. Menor relevancia tienen las acciones que implican el papel de la familia.

Finalmente, se destaca el modelo diseñado por Seong y Ho (2012) para dinamizar tecnológicamente la escuela donde las tareas y funciones se distribuyen en la línea de:

- Aprovechar las reformas que plantean las políticas TIC para pensar estratégicamente y marcar puntos de referencia (bechmarks) que atraigan el compromiso de profesores y estudiantes.

- Brindar apoyo al profesorado en el diseño de sus clases con TIC y ofrecerles formación continua.

- Generar actitudes positivas y una mentalidad de cambio y aprendizaje continuo.

- Rediseñar el uso del tiempo, del espacio y de la estructura organizativa para apoyar el cambio.

- Rediseñar el currículum para incorporar el uso de las TIC.

- Estratégicamente utilizar a otros agentes para incrementar las posibilidades de ampliar la base de liderazgo para las TIC. En este sentido se podría dejar abierta la posibilidad de incorporar a las familias en el proceso de incorporación de las TIC (BECTA, 2010).

Para concluir, se añade que en una de las investigaciones desarrolladas por Tondeur, Coopert y Newhouset (2010) se destaca la idea de que el apoyo del coordinador TIC es 
un factor importante en favorecer la motivación de los agentes educativos implicados y de fomentar el uso de las TIC para el aprendizaje. Más específicamente, el éxito es más probable cuando el apoyo es impulsado por las necesidades específicas de los maestros. Algunas estrategias eficaces son el apoyo uno a uno, la creación de modelos de referencia y la colaboración y apoyo entre compañeros. También la literatura sugiere que el éxito de la integración de las TIC depende parcialmente del apoyo y la coordinación que se proporciona desde la escuela.

\section{REFERENCIAS BIBLIOGRÁFICAS}

Afshari, M., Bakar, K., Luan, W., \& Siraj, S. (2012). Factors affecting the transformational leadership role of principals in implementing ICT in schools. The Turkish Online Journal of Educational Technology, 11(4), 164-176.

Anderson, R.E., \& Dexter, S. (2005). School technology leadership: an empirical investigation of prevalence and effect. Educational Administration Quarterly, 41(1). 49-82.

Area, M. (2010). El proceso de integración y uso pedagógico de las TIC en los centros educativos. Un estudio de casos. Revista de Educación, 352, 77-97.

Balanskat, A., Blamire. R., \& Kefala, S. (2006). The ICT Impact Report: A Review of Studies of ICT Impact on Schools in Europe. European Schoonet.

BECTA. (2010). I'm stuck. Can you help me? A report into parents' involvement in school work at home. Coventry: BECTA.

De Pablos, J., Area, M., Valverde, J., \& Correa, J. M. (2010). Políticas educativas y buenas prácticas con TIC. Barcelona: Graó.

Devolder, A., Vanderlinde, R., van Braak, J., \& Tondeur, J. (2010). Identifying multiple roles of ICT coordinators. Computers \& Education, 55, 1651-1655.

Fernández, M. R., Sosa, M. J., \& Garrido, M. C. (2011). Retos para la figura de la coordinación TIC. REDEX, 1, 55-75.

Fernández, S., \& Lázaro, M. N. (2008). Coordinar/a TIC. Pieza clave para la integración de las nuevas tecnologías en las aulas. Revista Latinoamericana de Tecnología Educativa, 7(2), 177187.

Fishman, B. J., \& Zhang, B. H. (2003). Planning for technology: the link between intentions and use. Educational Technology, 43, 14-18.

González-Pérez, A. (2010). ¿Qué nos interesa evaluar de las políticas educativas TIC españolas? Revista Fuentes, 10, 206-220.

González-Pérez, A. (2012). Regional Educational ICT policies in Spain and their Effects in Schools. In Chova, L.G., Martinez, A.L., \& Torres, I.C. (Eds.), Proceedings Paper of 5th International Conference of Education, Research and Innovation (ICERI), Valencia.

González-Pérez, A. (2014a). A comprehensive model to assess policies for ICT in education. IDEE 14 Proceedings of the 2014 Workshop on Interaction Design in Educational Environments. ACM. New York, NY, USA. doi:10.1145/2643604.2643612

González-Pérez, A. (2014b). Characterization of inclusive practices in schools with Education technology. Procedia-Social and Behavioral Sciences, 132, 357-363. doi:10.1016/j. sbspro.2014.04.322

González-Pérez, A., \& De Pablos, J. (2015). Factores que dificultan la integración de las TIC en las aulas. Revista de Investigación Educativa, 33(2), 401-417. doi:10.6018/rie.33.2.198161

Hancock, V. E. (1990). Promoting secondary school computer use? A coordinator is the key. Paper presented at the meeting of the International Conference on Technology and Education, Brussels, Belgium. Recuperado desde http://eric.ed.gov/?id=ED327143. 
Lai, K. W., \& Pratt, K. (2004). Information and communication technology (ICT) in secondary schools: the role of the computer coordinator. British Journal of Educational Technology, 35(4), 461-475.

Lai, K.W., Trewern, A., \& Pratt, K. (2002). Computer coordinators as change agents: some New Zealand observations. Journal of Technology and Teacher Education, 10, 539-551.

Marcovitz, D. (2000). The roles of the computer coordinators in supporting technology in schools. Journal of Technology and Teacher Education, 8, 259-273.

Pérez, M. A., Aguaded, J.I., \& Fandos, M. (2010). Percepciones, retos y demandas de los directivos y coordinadores de los centros TIC andaluces. Educatio Siglo XXI, 28(1), 295-316.

Schiller, J. (2003). Working with ICT: Perceptions of Australian principals. Journal of Educational Administration, 41(2), 171-185. doi:10.1108/09578230310464675

Seong, D.N.F., \& Ho, J.M. (2012). How leadership for an ICT reform is distributed within a school. International Journal of Educational Management, 26(6), 529-549. doi: $10.1108 / 09513541211251370$

Strudler, N.B. (1994). The role of school-based technology coordinators as change agents in elementary school programs: A follow-up study. Paper presented at the annual meeting of the American Educational Research Association, New Orleans, LA. Recuperado desde http://eric. ed.gov/?id=ED381139

Strudler, N.B., Falba, C., \& Hearrington, D. (2001). The evolving role of computer-based technology coordinators in elementary programs. Paper presented at the meeting of the National Educational Computer Conference, Chicago, IL. Recuperado desde http://tl.unlv.edu/ strudler/necc01.pdf

Tearle, P. (2004). A theoretical and instrumental framework for implementing change in ICT in education. Cambridge Journal of Education, 34, 331-351.

Tondeur, J., Coopert, M., \& Newhouset, C. P. (2010). From ICT coordination to ICT integration: A longitudinal case study. Journal of Computer Assisted Learning, 26, 296-306. doi:10.1111/ j.1365-2729.2010.00351.x

Tondeur, J., van Braak, J., \& Valcke, M. (2007). Primary school curricula and the use of ICT in education. Two worlds apart? British Journal of Educational Technology, 38, 962-975.

Vanderlinde, R., Dexter, S., \& van Braak, J. (2012). School-based ICT policy plans in primary Education: Elements, typologies and underlying processes. British Journal of Educational Technology, 43(3), 505-519. doi:10.1111/j.1467-8535.2011.01191.x

Watson, D. (2006). Understanding the relationship between ICT and education means exploring innovation and change. Education and Information Technologies, 11, 199-216.

Zhao, Y., \& Conway, P. (2001). What's in, what's out: an analysis of state educational technology plans. Teachers College Record. Recuperado desde http://www.tcrecord.org 
